\title{
Pseudomonas infections in cystic fibrosis
}

Pseudomonas aeruginosa is the main pathogen of the genus pseudomonas in cystic fibrosis, although Pseudomonas cepacia has been increasingly isolated from patients with cystic fibrosis in recent years. This article gives an overview of the microbiological factors that account for their pathogenicity. Colonisation, progression to chest infection, and management are also reviewed.

\section{Microbiology of Pseudomonas aeruginosa}

In the laboratory $P$. aeruginosa can grow in the presence of a wide variety of substrates and will even multiply in distilled water and weak disinfectants. This great adaptability is also reflected in its expression of certain virulence factors in response to environmental conditions. The use of classical genetic techniques (chemical mutagenesis, transduction, and conjugation) has helped in the characterisation of its virulence factors, and the recent introduction of recombinant deoxyribonucleic acid techniques should increase our understanding of pseudomonas infections, which range from acute septicaemia, as in patients with burns, to chronic infection in patients with cystic fibrosis.

The most striking difference between strains of $P$. aeruginosa isolated from patients with cystic fibrosis and those isolated from patients with other conditions is the production of an exopolysaccharide $\beta 1,4-$ linked mannuronic acid and L-guluronic acid, usually referred to as alginate; this exopolysaccharide is readily recognised in the laboratory because the colonies have a mucoid appearance. In vitro, production of alginate is an unstable characteristic, but mucoid variants are selected in vivo. Alginate protects the organism from opsonisation and phagocytosis ${ }^{1}$ and forms an elastic gel, inside which microcolonies develop, in the presence of high calcium ion concentrates such as occur in the lungs of patients with cystic fibrosis. Alginate also contributes to virulence by enhancing colonisation by mucoid strains ${ }^{2}$ and it has recently been proposed that alginate contributes to lung damage due to the formation of immune complexes. ${ }^{3}$

$P$. aeruginosa produces several extracellular virulence factors, the most toxic of which is exotoxin A. Exotoxin $\mathrm{A}$ is toxic for human macrophages, which may assist the survival of the organism and have been shown to contribute to chronic lung infection in rats. An inverse relation between anti-exotoxin A antibodies and clinical scores, in patients with cystic fibrosis, also suggests that exotoxin A contributes to infection. ${ }^{4} \mathrm{~A}$ recent study showed that $100 \%$ of strains of $P$. aeruginosa from patients with cystic fibrosis produce phospholipase $\mathrm{C}^{5}{ }^{5}$ this acts preferentially against phosphatidylcholine, which is the major component of lung surfactant. $P$. aeruginosa also produces several proteases, including an elastase, which contribute to local tissue damage; recent studies have shown that these inhibit neutrophil chemotaxis and the $\mathrm{C}_{3}$ and $\mathrm{C}_{5}$ a components of complement, and break down IgA. ${ }^{6}$

\section{Colonisation by $P$. aeruginosa and progression to disease}

Colonisation of the respiratory tract by $P$. aeruginos $a$ is encountered in $17-90 \%$ of patients attending cystic fibrosis clinics; ${ }^{7}$ rates increase with age, the mean age of onset being 9-10 years. ${ }^{8}$ Most patients initially experience intermittent colonisation with non-mucoid forms, which subsequently become mucoid and intractable; it should be noted that all strains genetically code for production of alginate, but a suppressor gene prevents expression of this characteristic in non-mucoid forms.

Initially, the patient may produce little or no sputum and organisms may be scanty, which makes detection of colonisation difficult. Brett et al have recently shown that patients with cystic fibrosis develop IgG antibodies, detectable by enzyme linked immunosorbent assay (ELISA), to $P$. aeruginosa surface antigens shortly before or within one or two months after colonisation. ${ }^{9}$ The persistent isolation of mucoid $P$. aeruginosa is associated with clinical deterioration, ${ }^{10}$ and Brett et al have shown a correlation between the immunological changes and the severity of the infection. ${ }^{11}$ Currently, most patients with cystic fibrosis who die with pulmonary complications are infected with mucoid forms of $P$. aeruginosa.

\section{Treatment of $P$. aeruginosa}

Antipseudomonal penicillins combined with an aminoglycoside antibiotic, in high dosage and together with physiotherapy, have been the mainstay of treatment in most centres. While this leads to improved well being and lung function, eradication of the organism is uncommon and transient. More 
recently, intravenous ceftazidime, or imipenem and cilastatin in combination, or oral quinolones (nalidixic acid derivatives) have been tried. Subsequently, the patient's clinical condition usually deteriorates again over a period of three to four months.

Regular two week courses of antibiotics every three months have been shown to improve the five year survival, ${ }^{8}$ but many centres relate the timing of courses to clinical state. In one study $P$. aeruginosa was eliminated by antibiotics from half the patients who were treated within six months of developing IgG antibodies to the organism, ${ }^{9}$ as detected by ELISA. It remains to be seen if other workers will have similar experiences and whether this approach will have a major impact on long term survival. Long term treatment with nebulised antibiotic was shown by Hodson et $a^{12}$ and in a number of other studies to reduce the number of subsequent admissions to hospital and improve well being. Resistance and allergy sometimes develop, however, and the selection of other organisms, especially $P$. cepacia, is another possibility.

Resistance to antipseudomonal penicillins and aminoglycosides is increasing and in one centre resistance rates to carbenicillin, piperacillin, and gentamicin have been reported to be $70 \%, 30 \%$, and $40 \%$, respectively. Despite their short clinical use, resistance to ceftazidime, imipenem/cilastatin, and the quinolones has also occurred.

\section{Microbiology of $\boldsymbol{P}$. cepacia}

$P$. cepacia, although less closely related to $P$. aeruginosa than many pseudomonads, shares its versatility for growth and survival. Study of its virulence factors is only in its infancy. Apart from the production of proteases and lipases by most isolates from patients with cystic fibrosis, ${ }^{13}$ little else is clear.

\section{$P$. cepacia infection}

The number of centres that have reported this organism is increasing and one centre in the United States reported that $20 \%$ of their patients were colonised in 1983, with an annual rate of acquisition of $8 \% .^{14}$ Overall, the scale of the problem is substantially less, many centres reporting few or no cases. Many patients who become colonised show no change in their natural history, but some show an accelerated clinical deterioration and a few experience a fulminant infection with severe necrotising pneumonia demonstrable at necropsy. In reviewing the published works, Goldman and Klinger ${ }^{13}$ drew attention to the finding in one centre that $42 \%$ of patients with cystic fibrosis who died were colonised with $P$. cepacia. Treatment of the infections is difficult as the organism is inherently resistant to antipseudomonal penicillins, aminoglycosides, and colistin, but clinical improvement has been reported in some cases treated with ceftazidime.

Cross infection with the organism does occur and current therapeutic regimens for $P$. aeruginosa probably exert a selective pressure for this organism. While much of our attention is focused on $P$. aeruginosa, it is clearly important to study $P$. cepacia in depth.

\section{References}

1 Baltimore RS, Mitchell M. Immunologic investigations of mucoid strains of Pseudomonas aeruginosa: comparison of susceptibility to opsonic antibody in mucoid and nonmucoid strains. J Infect Dis 1980;141:238-42.

2 Marcus H, Baker NR. Quantitation of adherence of mucoid and nonmucoid Pseudomonas aeruginosa to hamster tracheal epithelium. Infect Immun 1985;47:723-9.

3 Pier GB. Pulmonary disease associated with Pseudomonas aeruginosa in cystic fibrosis: current status of the host-bacterium interaction. J Infect Dis 1985;151:575-80.

4 Jagger KS, Robinson DL, Franz ML, et al. Detection by enzyme-linked immunosorbent assays of antibody specific for Pseudomonas proteases and exotoxin A in sera from cystic fibrosis patients. J Clin Microbiol 1982;15:1054-8.

5 Granstrom M, Ericsson A, Standvic B, et al. Relation between antibody response to Pseudomonas aeruginosa exoproteins and colonisation/infection in patients with cystic fibrosis. Acta Paediatr Scand 1984;73:772-7.

${ }^{6}$ Döring G, Obernesser HJ, Botzenhart K, et al. Proteases of Pseudomonas aeruginosa in patients with cystic fibrosis. $J$ Infect Dis 1983;147:744-50.

${ }^{7}$ Friend PA. Pulmonary infection in cystic fibrosis. $J$ Infect 1986;13:55-72.

× Szaff M, Høiby N, Flensborg EW. Frequent antibiotic therapy improves survival of cystic fibrosis patients with chronic Pseudomonas aeruginosa infection. Acta Paediatr Scand 1983;72:651-7.

9 Brett MM, Ghonheim ATM, Littlewood JM. Serum IgG antibodies in cystic fibrosis patients with early Pseudomonas aeruginosa infection. Arch Dis Child 1987;62:357-61.

11 Klinger JD. Thomassen MJ. Occurrence and antimicrobial susceptibility of Gram-negative nonfermentative bacilli in cystic fibrosis patients. Diagnostic Microbiology of Infectious Disease 1985;3:149-58.

"Brett MM, Ghonheim ATM, Littlewood JM. Serum antibodies to Pseudomonas aeruginosa in cystic fibrosis. Arch Dis Child 1986;61:1114-20.

12 Hodson ME, Penketh ARL, Batten JC. Aerosol carbenicillin and gentamicin treatment of Pseudomonas aeruginosa infection in patients with cystic fibrosis. Lancet 1981;ii:1137-9.

13 Goldman DA, Klinger JD. Pseudomonas cepacıa: bıology, mechanisms of virulence, epidemiology. J Pediatr 1986;105 (suppl):806-12.

14 Thomassen MJ, Demko CA, Stern RC, et al. Pseudomonas cepacia: decrease in colonization in patients with cystic fibrosis. Am Rev Respir Dis 1986;134:669-72.

R H GEORGE Microbiology Department, Children's Hospital, Ladywood Middleway, Ladywood, Birmingham B16 8ET 